\title{
TEST PARTICLE ENERGIZATION BY CURRENT SHEETS AND NONUNIFORM FIELDS IN MAGNETOHYDRODYNAMIC TURBULENCE
}

\author{
Pablo Dmitruk, W. H. Matthaeus, and N. Seenu \\ Bartol Research Institute, University of Delaware, Newark, DE 19716; pablo@bartol.udel.edu \\ Received 2004 July 28; accepted 2004 August 20
}

\begin{abstract}
We perform numerical experiments of test particle energization in turbulent magnetic and electric fields obtained from pseudospectral direct numerical solutions of compressible three-dimensional magnetohydrodynamic (MHD) equations with a strong background magnetic field. The natural tendency of turbulent MHD fields is to form current sheets along the magnetic field direction, as well as strong nonuniform fields in the transverse directions. By associating the MHD dissipation length scale with the ion inertial scale, we found differential energization in parallel and perpendicular directions according to the type of particles considered. Electrons develop large parallel velocities, especially in current sheets. Protons instead show higher perpendicular energization due to the nonuniform perpendicular induced electric field produced by the plasma MHD velocity, which varies on proton length scales. Implications for dissipation mechanisms in a coronal heating model are discussed.
\end{abstract}

Subject headings: acceleration of particles — MHD — turbulence

\section{INTRODUCTION}

Test particle simulations can be useful for gaining insight into the complex topic of bridging large-scale macroscopic magnetohydrodynamic (MHD) descriptions and particle kinetic physics descriptions. As such, they have been used in spatial diffusion studies, where usually turbulent MHD fields are modeled by giving their second-order spatial correlation functions or, equivalently, the energy spectrum. This is the area, for instance, of cosmic-ray scattering and transport (Giacalone \& Jokipii 1999). When studying momentum diffusion (stochastic acceleration), a typical approach is to represent the MHD fields as a collection of waves with prescribed dispersion relations between frequency and wavenumber vectors, given amplitudes, and random phases (Miller et al. 1997). This "wave turbulence" representation is used then to address wave-particle interactions. Another approach using test particles includes randomly generated (Gaussian) fields with (modeled) locally enhanced resistivity in current "hot spots" (Arzner \& Vlahos 2004).

In another very different set of models, instead of using spectral representations or waves to model turbulent fluctuations, the fields are considered in special geometries, with large-scale and coherent properties. Among those studies are the cases of magnetic reconnection and its influence on particle acceleration, for example in the magnetosphere or solar flares. Reconnecting fields for pushing test particles can be given analytically (Heerikuisen et al. 2002) or from MHD simulations, which can include driving flows (Schopper et al. 1999; Birn \& Hesse 1994) or initial-value problems with added perturbations (Ambrosiano et al. 1988). In all these examples, the main geometry of reconnection is set up at the beginning and is not the result of a natural evolution of the fields.

From the largely random prospective used in wave turbulence or stochastic acceleration studies to the more organized scenario of reconnection studies, there is, however, an intermediate class of problems that, in our view, has received very little attention. This corresponds to problems in which turbulence results as a natural evolution of the MHD fields (and so is not modeled) and, at the same time, reconnecting-like geometries appear as an outcome of that evolution.
We are quite aware of the limitations of the direct approach for the MHD fields that we are attempting to use, in terms of the length- and timescales available with actual computer resources; however, we believe that it is worthwhile to explore this issue, even with these limitations in place. In this regard, the approach based on modeling turbulent fields through spectra and waves has the advantage of achieving in principle very high spatial resolution up to particle scales. However, it lacks an important property of turbulence, which is the fact that MHD fields tend to form coherent and singular structures (like current sheets) that are completely absent in the wave turbulence picture. It is possible that for scattering or transport problems this is not relevant, but it could be very important for particle energization problems, especially on short timescales.

The approach that we follow is perhaps closer to the reconnecting geometry test particle studies, since, as we state below, the fields that we consider are not going to be evolved in time (so there is no possibility at all of waves in the system). But an important distinction is that the fields that we consider are obtained from a direct numerical simulation of MHD turbulence, in which no initial reconnecting structure is set up to be driven or perturbed, but rather any coherent or singular structure formation results from the natural evolution of the fields. Once the system has evolved for a couple of dynamical times, the fields are saved for pushing the particles in the largely irregular (but not totally random) configuration obtained. The assumption here is that the effect of the time evolution of the fields is of secondary importance for the test particle behavior. This in particular is applicable for short times, where our study is focused. In this sense, the study is limited and we are not attempting to address the long-time momentum diffusion problem here. Limitations on the size of the simulation (and resolving scales) would also prevent a correct study of the diffusion problem.

The problem that we consider continues in the line of study that we started in Dmitruk et al. (2003) in an attempt to fill a gap in numerical studies of test particles in directly obtained turbulent fields with singular structure formation. Idealized and theoretically motivated as it is, it turns out that this problem could be of relevance for a long-standing problem in space physics, which is the energization of electrons and ions in the 
corona and solar wind (Kohl et al. 1997, 1998). The results presented here could be regarded as the onset of a direct dissipation mechanism for a coronal heating model based on development of MHD turbulence and current sheets (Dmitruk \& Matthaeus 2003). Kinetic dissipation mechanisms for coronal heating based on well-studied wave-particle energization models, such as ion cyclotron resonance (see Hollweg \& Isenberg 2002 and references therein), or kinetic Alfvén waves models (Cranmer \& van Ballegooijen 2003; Gary \& Nishimura 2004) should also be mentioned.

The organization of the paper is as follows. In $\S 2$ we describe the equations and properties of the MHD fields, including the formation of current sheets. In $\S 3$ results are given for different types of test particles, "electrons" and "protons," moving in these fields and described by the nonrelativistic equations of motion. A qualitative picture of the different type of energization for each kind of particle is discussed. Section 4 discuss mostly speculative ideas about how these results could fit into a coronal heating model. Section 5 contains the conclusions.

\section{THE FIELDS}

\subsection{MHD Definitions and Equations}

The macroscopic description of the plasma is given by compressible three-dimensional MHD, which involves a fluctuating flow velocity $\boldsymbol{v}(x, y, z, t)$, magnetic field $\boldsymbol{b}(x, y, z, t)$, and density $\rho(x, y, z, t)$. We assume the presence of a large-scale magnetic field (DC field) in the $z$-direction, $\boldsymbol{B}_{0}=B_{0} \hat{z}$, so the total magnetic field is $\boldsymbol{B}=\boldsymbol{B}_{0}+\boldsymbol{b}$.

The MHD equations are

$$
\begin{gathered}
\frac{\partial \boldsymbol{v}}{\partial t}+\boldsymbol{v} \cdot \nabla \boldsymbol{v}=-\frac{1}{\rho} \nabla p+\frac{1}{4 \pi \rho} \boldsymbol{J} \times \boldsymbol{B}+\nu\left(\nabla^{2} \boldsymbol{v}+\frac{1}{3} \nabla \nabla \cdot \boldsymbol{v}\right) \\
\frac{\partial \boldsymbol{B}}{\partial t}=\nabla \times(\boldsymbol{v} \times \boldsymbol{B})+\mu \nabla^{2} \boldsymbol{B}
\end{gathered}
$$

with $p$ the pressure, $\nu$ the viscosity, $\mu$ the magnetic diffusivity, and $\boldsymbol{J}=\nabla \times \boldsymbol{B}$ the current density (here $\boldsymbol{J}=4 \pi \boldsymbol{j} / c$, where $\boldsymbol{j}$ is the current density in Gaussian units and $c$ is the speed of light). A continuity equation for $\rho$ and an equation of state complete the system. We assume a nearly incompressible regime with Mach number 0.25 and treat the pressure as polytropic, $p \sim \rho^{5 / 3}$.

We consider periodic boundary conditions in a cube of side $L_{\text {box }}=2 \pi L$. The value of $L$ is of the same order as the energycontaining scale or the magnetic field correlation length, which is approximately constant throughout the short time evolution of the system. The periodic box contains then about six correlation scales, $L_{\mathrm{box}}=2 \pi L$.

The initial magnetic and velocity fluctuation root mean square (rms) values are $\delta B=\left\langle|\boldsymbol{b}|^{2}\right\rangle^{1 / 2}$ and $\delta v=\left\langle|\boldsymbol{v}|^{2}\right\rangle^{1 / 2}$, and we set up $\delta v=\delta B /\left(4 \pi \rho_{0}\right)^{1 / 2}=v_{0}$, which is the plasma Alfvénic speed based on the fluctuations amplitude $\left(\rho_{0}\right.$ is the mean density). Note that another (usually much larger) Alfvén speed can also be defined for the DC field part, $V_{\mathrm{A}}=B_{0} /\left(4 \pi \rho_{0}\right)^{1 / 2}$. This is the speed of Alfvén waves propagating parallel to the DC field, but it has no relation to the turbulent fluctuations amplitude. The kinetic and magnetic Reynolds numbers are $R=v_{0} L / \nu$ and $R_{m}=v_{0} L / \mu$, which measure the strength of the nonlinear terms in the MHD equations compared to the dissipative terms. We take $R=R_{m}=1000$, which are limited here by the available spatial resolution.

The timescale for the turbulent MHD fields is $t_{0}=L / v_{0}$ (eddy turnover time). We consider a decaying simulation from an initial state with the kinetic and magnetic field fluctuations populating an annulus in Fourier $k$-space such that $1 \leq k \leq 4$, with constant amplitude and random phases. We employ a pseudospectral code with resolution of $256^{3}$ Fourier modes, which guarantees that dissipation scales $l_{d}>2 \pi L / 256$ are fully resolved for the moderate Reynolds numbers considered. After $2 t_{0}$ times, a turbulent state with a range of scales from $L$ to $l_{d} \approx L / 32$ has been developed (recall here that some room has been made in the simulation box to contain about six energy-containing scales $L$, so about 2 orders of magnitude in $k$-space are available). We then take a snapshot of the magnetic, velocity, and current density fields at that time to be used for pushing the test particles.

\subsection{MHD Field Structure}

The structure of the magnetic field and the current density $J_{z}$ in the parallel direction can be seen in Figure 1. In the left panel we show a cross section in the plane $x-z$ of the current density $J_{z}$ in color tones. Yellow (light) corresponds to strong positive values, while blue (dark) corresponds to strong negative values. Superposed with arrows is a magnetic field vector constructed with the components $b_{x} \hat{\boldsymbol{x}}+\left(b_{z}+B_{0}\right) \hat{z}$ in the same cross section. The presence of the DC field is clear. The ratio between the DC field and the rms of magnetic fluctuations at this time is $B_{0} / \delta B=10$. This value is fixed for the test particle simulations. Current density structures preferentially aligned along the DC field direction can be seen in this panel. This is a manifestation of the well-known anisotropic behavior of magnetic field fluctuations in MHD with a DC field (Shebalin et al. 1983; Oughton et al. 1994). In this case, the current density is directly related to small-scale fluctuations of the magnetic field, and so the anisotropy at these scales is clearly visible. The right panel shows the current density $J_{z}$ in an $x-y$ cross section and the perpendicular magnetic field vector $b_{x} \hat{\boldsymbol{x}}+b_{y} \hat{\boldsymbol{y}}$ in the same section, with arrows superposed (note that the arrows here are not exactly in scale with the left panel arrows, because of the small ratio 1/10 between them). The current density structures are much more varying in the perpendicular cross section, confirming the anisotropy.

The overall picture is that current sheet structures along the DC field are formed as a natural evolution of the turbulent MHD fields. The magnetic field fluctuations in the perpendicular plane are also rapidly varying in space. These structures resemble two-dimensional reconnecting structures (Matthaeus \& Lamkin 1986; Biskamp \& Welter 1989), although since this is three-dimensional, they should be better compared with the type known as "component reconnection," which is the two-dimensional reconnection of the perpendicular component of a magnetic field with a strong guide field (Birn et al. 1989). However, the plots show that the form of the magnetic field is not quite the ideal type described commonly in reconnection theory, but rather a more complex and fluctuating picture. Moreover, perpendicular currents $J_{x}, J_{y}$ are also formed (not shown); however, they lack the coherent structure seen for $J_{z}$.

In Figure 2 the velocity field is shown. In the left panel the velocity field vector constructed with components $v_{x} \hat{\boldsymbol{x}}+v_{z} \hat{z}$ is shown as arrows over the current density in color tones as before. The current density structures are shown here for reference with the previous figure. The right panel shows the 

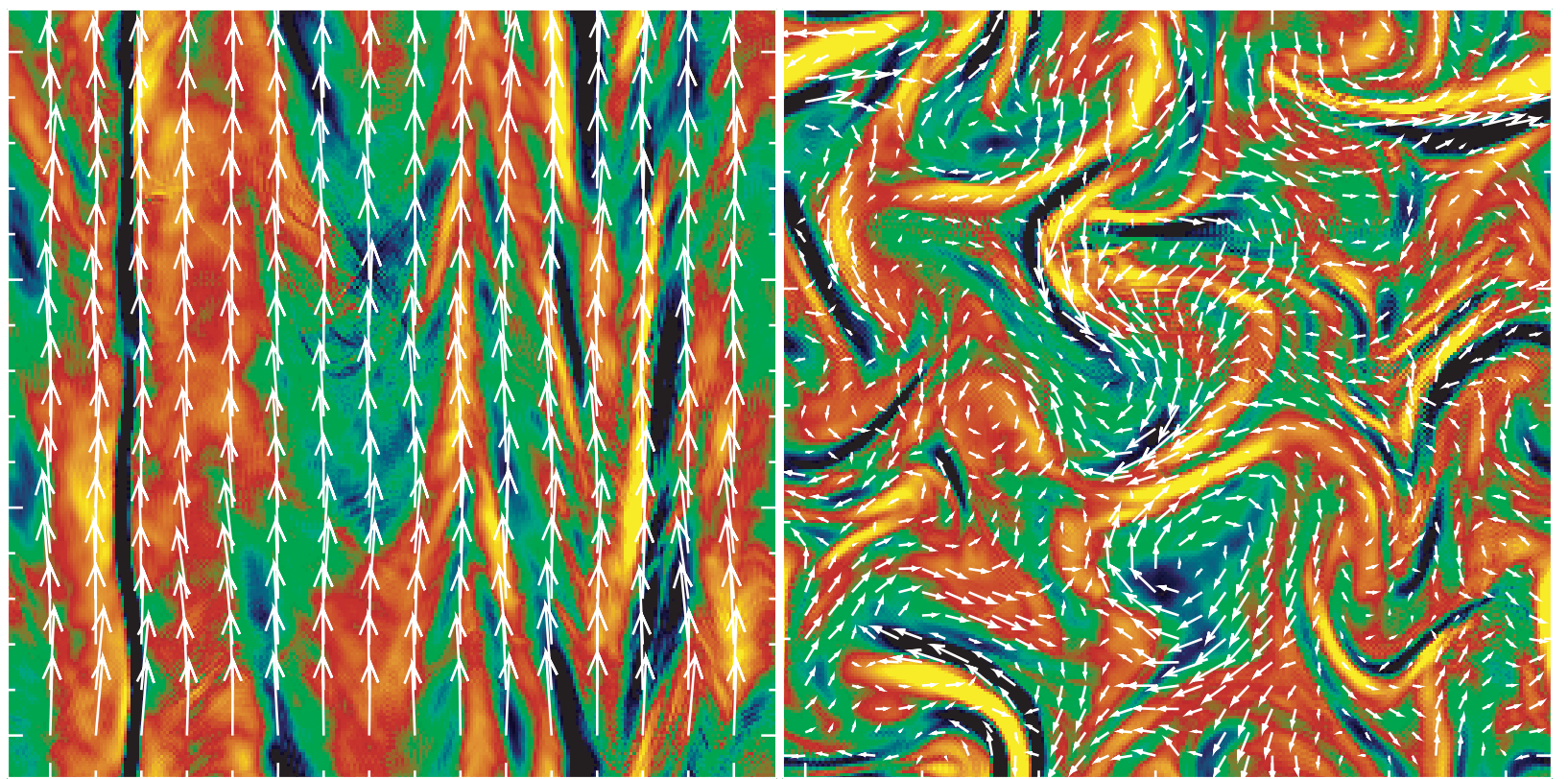

FIg. 1. Left: Cross section in a plane $x-z$ of the current density $J_{z}$ in color tones. Yellow (light) is positive $J_{z}$, blue (dark) is negative $J_{z}$. Superposed with arrows is a vector magnetic field constructed with $b_{x}$ and $b_{z}+B_{0}$ components, where $B_{0}$ is the background magnetic field in the $z$-direction. Right: Cross section in a plane $x-y$ of the current density $J_{z}$ and superposed arrows for the vector magnetic field constructed with $b_{x}$ and $b_{y}$ components.

velocity field vector in the perpendicular plane $v_{x} \hat{\boldsymbol{x}}+v_{y} \hat{\boldsymbol{y}}$. As with the magnetic field fluctuations, the velocity field fluctuations are highly varying in this plane. As will become clear later, these variations are important for the particles' energization.

With the magnetic field, velocity, and current density, the electric field can be obtained through Ohm's law,

$$
\boldsymbol{E}=-\frac{1}{c} \boldsymbol{v} \times \boldsymbol{B}+\frac{v_{0} L}{R_{m} c} \boldsymbol{J}
$$

and is the result of the electromotive force (EMF) term due to the plasma motion plus a formally small resistive term that is important in current sheet regions (here we write the magnetic diffusivity as $\mu=v_{0} L / R_{m}$ ).

\section{TEST PARTICLES}

The nonrelativistic equations of motion for the charged particles in the fields obtained from MHD (in cgs units) are

$$
\frac{d \boldsymbol{u}}{d t}=\frac{q}{m}\left(\frac{1}{c} \boldsymbol{u} \times \boldsymbol{B}+\boldsymbol{E}\right), \quad \frac{d \boldsymbol{x}}{d t}=\boldsymbol{u},
$$

where $\boldsymbol{u}$ is the particle velocity, $\boldsymbol{x}$ the position, $q$ the charge, and $m$ the mass.
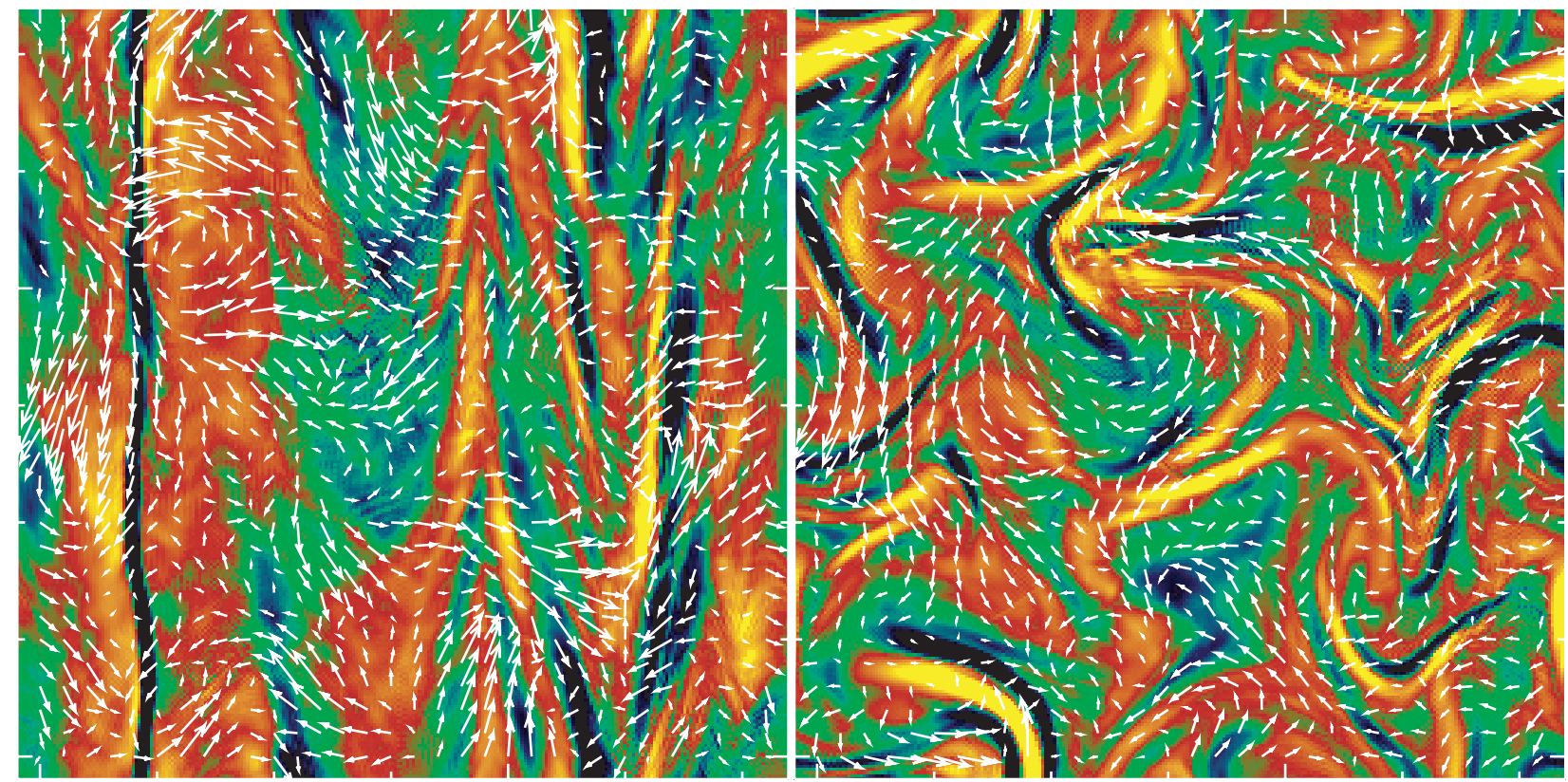

FIG. 2. - Left: Cross section in a plane $x-z$ of the current density $J_{z}$ in color tones. Superposed with arrows is a velocity field constructed with $v_{x}$ and $v_{z}$ components. Right: Cross section in a plane $x-y$ of the current density $J_{z}$ and superposed arrows for the velocity field constructed with $v_{x}$ and $v_{y}$ components. 
A fast timescale is given here by the inverse of the particle gyrofrequency $w_{g}=q B /(\mathrm{mc})$ around a magnetic field of intensity $B$, and a small length scale (unless the particle energy is very high) is given by the gyroradius $r_{g}=u_{\perp} / w_{g}=$ $u_{\perp} m c /(q B)$, where $u_{\perp}$ is the velocity of the particle in the perpendicular plane to the magnetic field. A nominal gyroradius can be defined by setting $u_{\perp}=v_{0}$ (i.e., the typical plasma speed) and $B=B_{0}$; thus, $r_{0}=v_{0} m c /\left(q B_{0}\right)$. Recalling here that $v_{0}=\delta B /(4 \pi \rho)^{1 / 2}$, where $\rho$ is the plasma density, and introducing the ion (proton) inertial scale $\rho_{i i}=m_{p} c /\left(e^{2} 4 \pi \rho\right)^{1 / 2}$, with $m_{p}$ the proton mass and $e$ the charge, we have $r_{0}=$ $Z\left(m / m_{p}\right)\left(\delta B / B_{0}\right) \rho_{i i}$, where we use $q=Z e$. A dimensionless quantity can be constructed by comparing this small particle length scale $r_{0}$ with the large MHD scale $L$,

$$
\frac{r_{0}}{L}=Z \frac{m}{m_{p}} \frac{\delta B}{B_{0}} \frac{\rho_{i i}}{L}
$$

A wide range of values of $r_{0} / L$ can be obtained, either by a change in the particle properties (charge, mass) or by a change in the ratio $\rho_{i i} / L$ between the ion inertial scale and the energycontaining scale. In general, we might expect that $\rho_{i i} / L \ll 1$. This is especially true for space physics and astrophysics applications. For instance, in the solar wind it can be estimated that $\rho_{i i} / L \sim 10^{-5}$. In a coronal hole (at about one solar radius), $\rho_{i i} / L \sim 10^{-6}$. In fact, there are good indications from observations in the solar wind (Leamon et al. 1998) that the length scale $l_{d}$ at which dissipation effects become noticeable in MHD is of the order of the ion inertial scale, that is, $l_{d} \sim \rho_{i i}$.

Attempting to resolve this full range of scales in a threedimensional MHD simulation requires an enormous cost in terms of computing power at present standards. As we stated before (see previous section) we have set up $l_{d}=L / 32$ (corresponding to the current sheet thickness observed in Fig. 1), so the identification $l_{d}=\rho_{i i}$ gives a modest value of $\rho_{i i} / L=$ $1 / 32$, and we study the properties of "protons" and "electrons" in this situation (note that the box simulation size is then $\left.L_{\mathrm{box}}=2 \pi L \approx 200 \rho_{i i}\right)$.

These test particles start from rest at random positions in the box and will move in the electromagnetic field obtained from the MHD simulation. We consider 50,000 particles that are moved using a Runge-Kutta fourth-order time integration method, with an adaptive time step calculation. The values of the fields at each particle position are obtained by linear interpolation in space from the grid of the MHD simulation.

When the rms displacement of the particles is about an energy-containing scale $L$, the simulation is stopped. Since we set the energy-containing scale 6 times smaller than the box size, this prevents effects of the periodic boundary conditions on the result. In this sense, our study is focused on the shorttime behavior of the particle velocity distribution and does not attempt to address the long-time transport problem. A direct numerical simulation of the MHD fields for a diffusion study would require a larger box size compared to the energycontaining scale, thus increasing even more the computational requirements. Otherwise, turbulence is usually modeled through second-order correlations (spectra) and random phases, and a wide range of scales is available. As we pointed out above, that approach lacks the dynamical formation of singular structures that naturally occurs in MHD. Fokker-Planck descriptions (Hall \& Sturrock 1967; Achatz et al. 1991; Schlickeiser \& Miller 1998) of the particle distribution evolution are possible in that approach, but that analytical level is not available here.
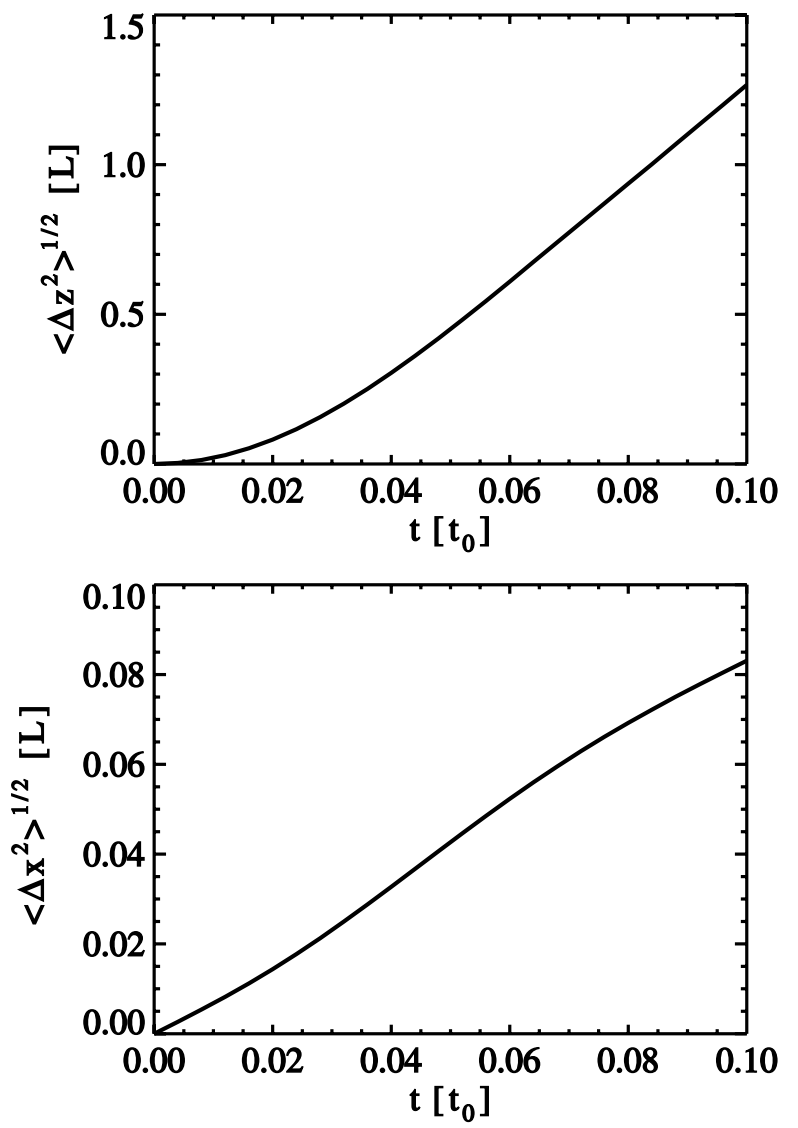

FIG. 3.-Top: The rms displacement of electrons as a function of time in the $z$-direction (parallel to the background magnetic field) $\left\langle\Delta z^{2}\right\rangle^{1 / 2}$. Bottom: The rms displacement in a transverse direction $\left\langle\Delta x^{2}\right\rangle^{1 / 2}$.

\subsection{Electrons}

Considering $m=m_{e}=m_{p} / 1836, Z=-1, \rho_{i i} / L=1 / 32$, and $\delta B / B_{0}=1 / 10$, then $r_{0}^{e} / L \approx 1.7 \times 10^{-6}$. Here $r_{0}^{e}$ is the previously defined nominal gyroradius $r_{0}$, for the particular case of electrons.

In Figure 3 the rms displacement of electrons as a function of time is shown. The top panel shows the rms displacement in the $z$-direction (parallel to the background magnetic field) $\left\langle\Delta z^{2}\right\rangle^{1 / 2}$ until it reaches a value of about 1 (i.e., an MHD energy-containing scale $L$ ); the bottom panel shows the rms displacement in a transverse direction $x$ (there is no difference on which transverse direction is chosen, $x$ or $y)\left\langle\Delta x^{2}\right\rangle^{1 / 2}$. It is clear from these plots that a large displacement of electrons occurs in the parallel direction as compared to the relatively small displacement observed in the transverse direction. The timescale at which most electrons have traveled a distance $L$ in the parallel direction is about $t_{\|} \sim 0.09 t_{0}$. This is a short timescale in terms of the plasma dynamic time and also (as we will show) short compared to the time it takes for protons to travel a distance $L$.

By that timescale, however, as can be seen in the top panel of Figure 4 a high parallel velocity has been developed by electrons. The rms of the parallel velocity is $\left\langle\Delta u_{z}^{2}\right\rangle^{1 / 2} \approx 19 v_{0}$, so it is many times the plasma MHD speed $v_{0}$. By comparison the rms value of a transverse component of the electron velocity is much smaller, $\left\langle\Delta u_{x}^{2}\right\rangle^{1 / 2} \approx 1.6 v_{0}$. The bottom panel in Figure 4 shows the ratio between the mean squares of the parallel and a transverse velocity, which reaches values as high as 150 . This implies the same high ratio for parallel to 

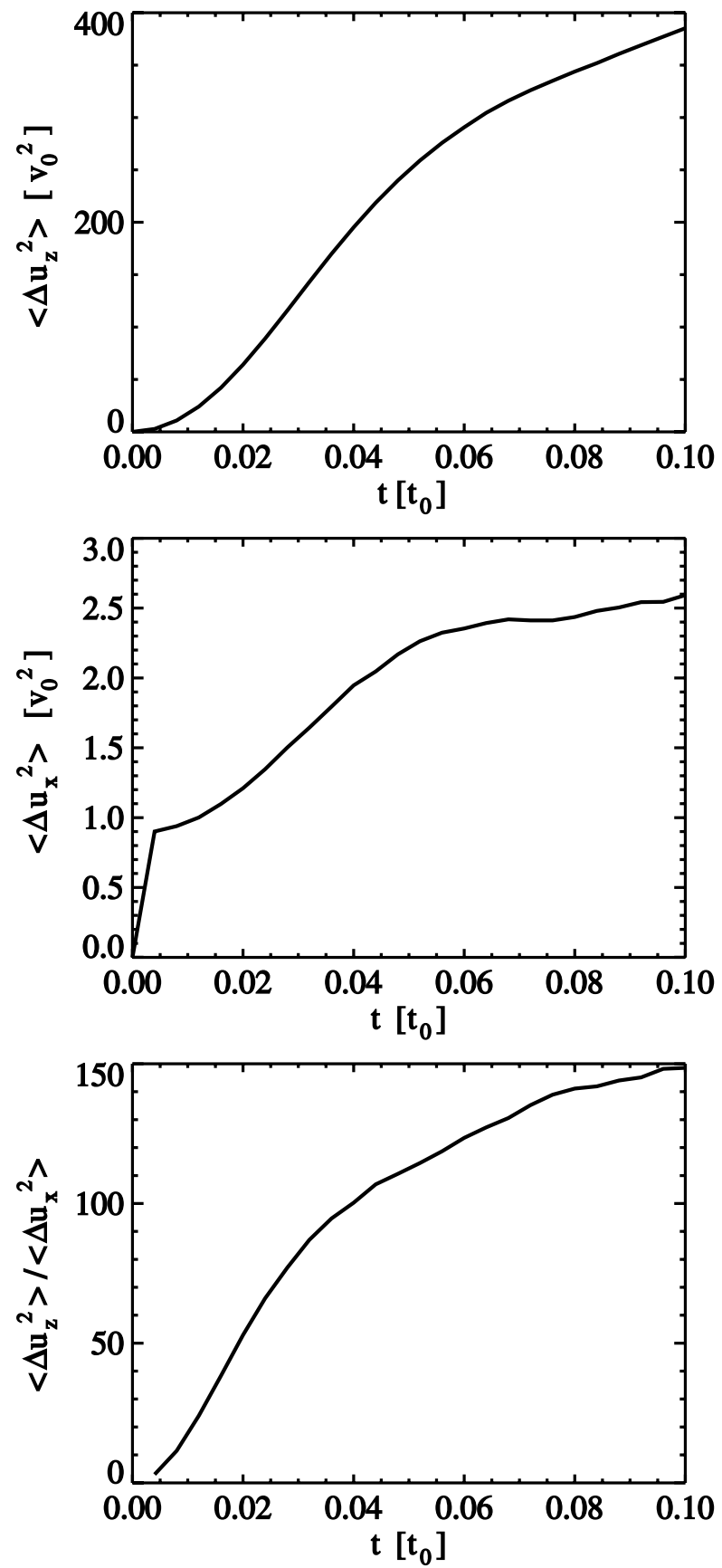

FIG. 4.-Top: Mean square velocity of electrons as a function of time in the parallel direction $\left\langle\Delta u_{z}^{2}\right\rangle$. Middle: Mean square velocity in a transverse direction $\left\langle\Delta u_{x}^{2}\right\rangle$. Bottom: Ratio of parallel to transverse mean square velocities $\left\langle\Delta u_{z}^{2}\right\rangle /\left\langle\Delta u_{x}^{2}\right\rangle$.

perpendicular electron temperatures, if defined as proportional to the mean square velocity.

A more complete behavior of the test electrons can be obtained by looking at their velocity distribution function at different times. This is shown in Figure 5. In these panels, the continuous line corresponds to the normalized distribution of parallel velocities $u_{j}=u_{z}$ while the dashed line corresponds to the distribution of a transverse velocity $u_{j}=u_{x}$. The top panel shows the distributions at a very short time, $t=4 \times 10^{-5} t_{0}$. This time can be expressed in terms of the electron gyroperiod in the strong magnetic field $B_{0}$, which is $\tau_{e}=2 \pi m_{e} c /\left(e B_{0}\right)=$ $2 \pi\left(r_{0}^{e} / L\right) t_{0} \approx 10^{-5} t_{0}$ (using the above chosen values for the electrons), so that for this panel $t \approx 4 \tau_{e}$. At this very short
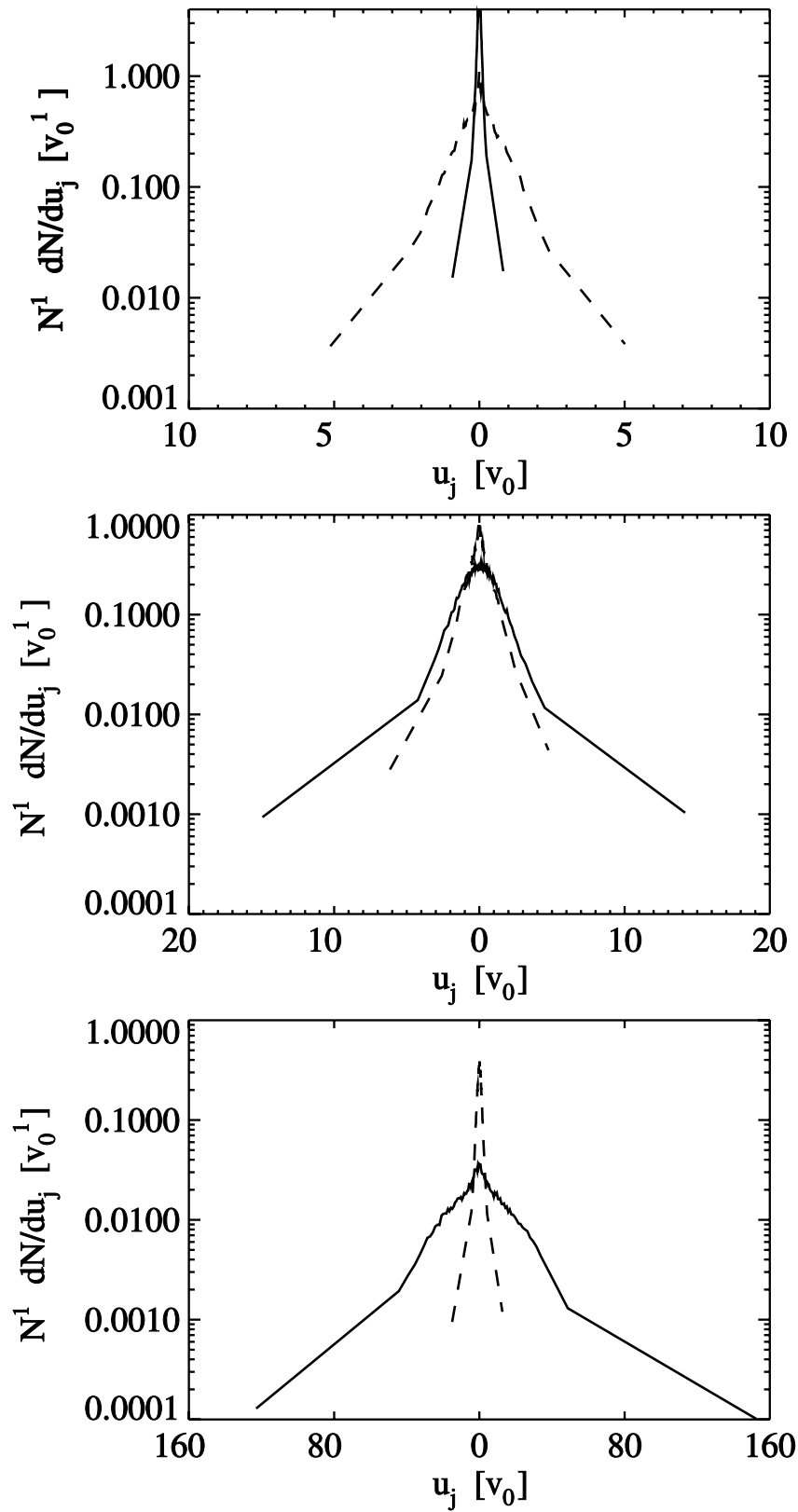

FIG. 5.-Velocity distribution functions of electrons at different times. The continuous line is the distribution of velocities in the parallel direction, and the dashed line is the distribution of velocities in a transverse direction. Top: Distributions at $t=4 \tau_{e}=10^{-5} t_{0}$. Middle: Distributions at $t=400 \tau_{e}=4 \times 10^{-3} t_{0}$. Bottom: Distributions at $t=10^{4} \tau_{e}=0.09 t_{0}$. Here $\tau_{e}$ is the electron gyroperiod and $t_{0}$ is the MHD timescale.

timescale transverse velocities of the particles are larger than the parallel velocities, and the values correspond approximately to values of the order of the plasma MHD speed $v_{0}$. That is, test electrons have rapidly acquired a transverse velocity of the order of the plasma MHD speed. The plot in the middle panel corresponds to the distributions at $t=4 \times 10^{-3} t_{0} \approx$ $400 \tau_{e}$. By this time, the parallel velocity of test electrons is already larger than the transverse velocity, both at the core part of the distributions and in the large developed tails at both positive and negative values. This behavior is further developed in time, as shown in the plot in the bottom panel, corresponding to the distributions at $t=0.09 t_{0} \approx 10^{4} \tau_{e}$. Both very large parallel and very large transverse velocities have developed by this time (note the different scale used in each 

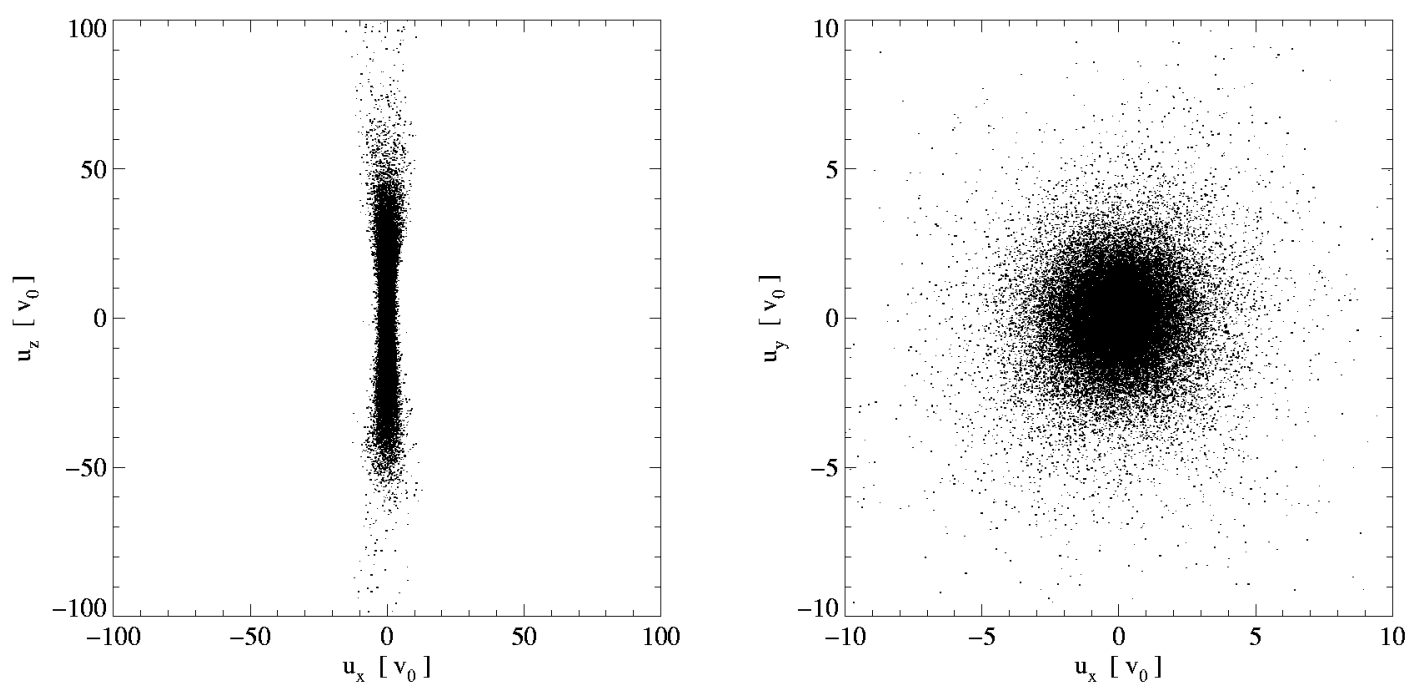

FIG. 6. - Left: Scatter plot of transverse $\left(u_{x}\right)$ and parallel $\left(u_{z}\right)$ velocity of electrons. Right: Scatter plot of transverse velocities $\left(u_{x}, u_{y}\right)$.

panel), but much larger parallel velocities both in the core and tails of the distributions are observed. Values as high as $u_{z}=$ $160 v_{0}$ have developed. The high parallel velocity tails are reminiscent of the observed strahl effect in solar wind energetic particle distributions (Gosling et al. 2004). Recall here that the $u_{z}$ component of the particle velocity is proportional to the (global) pitch angle $\mu=\boldsymbol{u} \cdot \boldsymbol{B}_{0} \propto u_{z}$.

The anisotropic behavior of the electron velocities is clearly shown in Figure 6. The left panel is a scatter plot of $u_{x}$ and $u_{z}$ electrons velocities, while the right panel shows a scatter plot of transverse velocities $u_{x}$ and $u_{y}$, which is clearly isotropic.

The behavior of the electrons in the situation that we have considered can be qualitatively explained by simple arguments. We are not attempting to explain details of the form of the distribution functions, but rather to provide a general picture of the differential energization that takes place for the electrons. In order to do this we write the equations for the velocity change of particles in the transverse and parallel directions. We first write the equations of motion of particles using equation (3) for the electric field $\boldsymbol{E}=-\boldsymbol{v} \times \boldsymbol{B} / \mathrm{c}+$ $\left(v_{0} L\right) /\left(R_{m} c\right) \boldsymbol{J}$ to obtain

$$
\frac{d \boldsymbol{u}}{d t}=\frac{q}{m c}\left[(\boldsymbol{u}-\boldsymbol{v}) \times \boldsymbol{B}+\frac{v_{0} L}{R_{m}} \boldsymbol{J}\right],
$$

where we recall that $\boldsymbol{u}$ is the particle velocity and $\boldsymbol{v}$ is the plasma velocity.

Now, separating into perpendicular and parallel components (and using $q=-e, m=m_{e}$ for electrons):

$$
\begin{gathered}
\frac{d \boldsymbol{u}_{\perp}}{d t}=-\frac{e}{m_{e} c}\left[\left(\boldsymbol{u}_{\perp}-\boldsymbol{v}_{\perp}\right) \times \boldsymbol{B}_{\|}+\left(\boldsymbol{u}_{\|}-\boldsymbol{v}_{\|}\right) \times \boldsymbol{b}_{\perp}+\frac{v_{0} L}{R_{m}} \boldsymbol{J}_{\perp}\right], \\
\frac{d \boldsymbol{u}_{\|}}{d t}=-\frac{e}{m_{e} c}\left[\left(\boldsymbol{u}_{\perp}-\boldsymbol{v}_{\perp}\right) \times \boldsymbol{b}_{\perp}+\frac{v_{0} L}{R_{m}} \boldsymbol{J}_{\|}\right]
\end{gathered}
$$

Let us assume $\boldsymbol{B}_{\|} \approx B_{0} \hat{\boldsymbol{z}}$, which neglects (or absorbs into the strong field $B_{0}$ ) the fluctuations in the parallel direction. On looking at the equation in the perpendicular direction, the first term on the right-hand side describes a perpendicular drift motion, with a velocity equal to the perpendicular plasma velocity $\boldsymbol{v}_{\perp}$ plus a motion around the guide field $B_{0}$. This happens on a very short timescale of the order of $\tau_{e}=$ $2 \pi\left(m_{e} c\right) /\left(e B_{0}\right)$, which as we have seen is much smaller than the plasma time $t_{0}$ (that is, $\tau_{e} \approx 10^{-5} t_{0}$ with the values used). In this short timescale the electrons acquire a perpendicular drift velocity of the order of the local plasma velocity $\boldsymbol{u}_{\perp} \approx \boldsymbol{v}_{\perp}$ plus the gyromotion (also with velocity amplitude of order $v_{\perp}$ if the particles started from rest or from a velocity close to the plasma speed $v_{0}$ ) around the strong magnetic field $B_{0}$. This very initial picture is supported by the top panel in Figure 5, which shows that a transverse velocity of the particles is already comparable to plasma speed values (the dispersion coming from the distribution of MHD velocities in the box, sampled by the particles' initial positions) in a very short time. Also, in Figure 4 for the mean square velocities, a sharp step from 0 to $v_{0}$ (the plasma speed) in a very short time can be seen in the panel plot corresponding to the transverse velocity of particles. Ignoring for the moment the effect of the second term on the right-hand side of equation (7) for the perpendicular velocity (either by assuming that fluctuations $\boldsymbol{b}_{\perp}$ are small or that parallel velocities are initially small, a situation that may change in time), we are left with a term involving perpendicular currents $\boldsymbol{J}_{\perp}$. This term may increase the perpendicular energy of the particles; however, these currents are very incoherent (as compared for instance with parallel currents), changing signs in distances much shorter than $L$ (the turbulent correlation length), so they do not produce a net effect on the particle energy.

We now look at equation (8) for the particle parallel velocity. The first term on the right-hand side involves the difference between the perpendicular velocities of the particle and the plasma. As we have seen, this is given by the gyromotion around the strong magnetic field $B_{0}$. For electrons, this is an oscillatory motion on a very fast timescale and on a very short length scale (gyroradius). Electrons will essentially see the same field $\boldsymbol{b}_{\perp}$ over many gyroperiods, so this term does not produce a net increase in the energy of the particles.

Where does the observed increase in the parallel energy of the electrons come from? The second term in equation (8) for the particle parallel velocity involves the current density in the parallel direction. As we have seen in the section on the MHD fields, the current is organized in sheet-like structures, being very coherent along the direction of the strong magnetic field. Although the ohmic current term can be very small compared 
to the induced EMF term in the electric field seen by the particles (due to the small factor $1 / R_{m}$ ), it can still be the relevant term in those sheet structures. Moreover, we have seen that the induced EMF term can be assimilated into the perpendicular drift motion of the particles. The ohmic current term instead remains coherent for long distances and is not damped by the strong magnetic field, which is aligned in the same direction as the formed currents. If electrons are moving on a short gyroradius (see Fig. 8 for that), they will essentially move along the strong current channels and increase their energy accordingly to an almost constant field over a correlation length in the parallel direction (of order $L$, the turbulence correlation length).

In this scenario, electrons that find strong currents will energize most, while electrons that encounter only average or small currents will only acquire a moderate energy. This process may continue until the electron leaves the box (or more precisely, travels about a correlation length distance). We can give an estimation of what those energies are by considering the electron parallel motion as described by

$$
\frac{d u_{\|}}{d t} \approx-\frac{e}{m_{e} c} \frac{v_{0} L}{R_{m}} J_{\|}, \quad \frac{d z}{d t}=u_{\|} .
$$

If we assume an approximately constant current $J_{\|}$, the distance traveled in the parallel direction in a time $t$ is $\Delta z \approx$ $J_{\|} t^{2} e v_{0} L /\left(2 m_{e} c R_{m}\right)$, so the time $t=t_{\|}$to move a correlation length $\Delta z \sim L$ is

$$
t_{\|}=\left(\frac{2 m_{e} c R_{m}}{e v_{0}\left|J_{\|}\right|}\right)^{1 / 2}
$$

and the parallel velocity acquired during that time is

$$
\Delta u_{\|}=\left(\frac{2 e v_{0}\left|J_{\|}\right|}{m_{e} c R_{m}}\right)^{1 / 2} L .
$$

The value of $J_{\|}$will depend on the location of the electron. If the particle finds a current channel, then $J_{\|} \approx J_{\|}^{\max }$; in contrast, if the particle is in an "average site," then $J_{\|} \approx \bar{J}_{\|}$, where the bar indicates a volume average. Using a reference current $J_{0}=\delta B / L$ and the definition of the ion inertial scale $\rho_{i i}$, we can rewrite equation (10) in terms of the unit time $t_{0}=L / v_{0}$, and equation (11) in terms of the unit velocity $v_{0}$, as

$$
\begin{aligned}
t_{\|} & =\left(\frac{2 m_{e}}{m_{p}} \frac{\rho_{i i}}{L} R_{m} \frac{J_{0}}{J_{\|}}\right)^{1 / 2} t_{0}, \\
\Delta u_{\|} & =\left(\frac{2 m_{p}}{m_{e}} \frac{L}{\rho_{i i}} \frac{1}{R_{m}} \frac{J_{\|}}{J_{0}}\right)^{1 / 2} v_{0} .
\end{aligned}
$$

For the MHD simulations that we performed, we observed a maximum value $J_{\|}^{\max } / J_{0}=47$. Replacing this value and the values that we considered for the test electron simulations (see above), we get the estimate $t_{\|} \approx 0.03 t_{0}$ and $\Delta u_{\|} \approx 74 v_{0}$. This estimate corresponds (in order of magnitude) to the tails of the velocity distribution function observed in Figure 5. Accordingly, with this estimation the electrons that acquire the higher parallel velocity will also travel a distance $L$ on a faster timescale than the other electrons. For electrons that move in "average currents," using the observed value in the MHD simulation of $\bar{J}_{\|} / J_{0}=4.5$ we obtain $t_{\|} \approx 0.09 t_{0}$ and $\Delta u_{\|} \approx$ $20 v_{0}$. The estimated parallel velocity corresponds to the core of the velocity distribution function shown in Figure 5. The

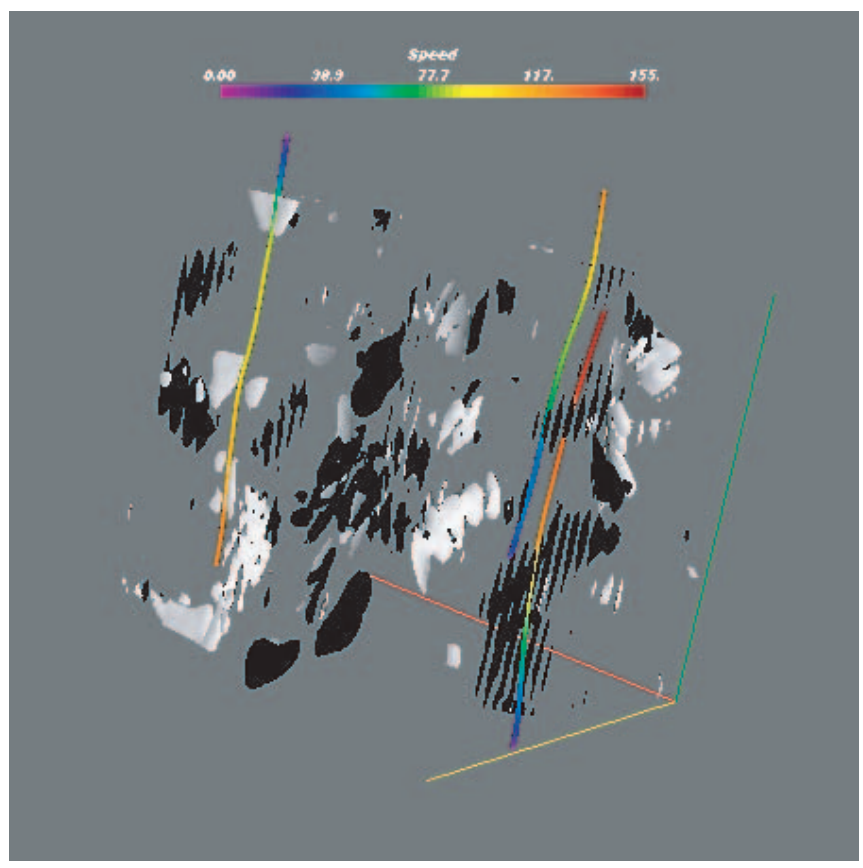

FIG. 7.-Trajectories of three of the most energetic electrons. The structures in the background are contour surfaces of the current density $J_{z}$, with white indicating strong positive currents and black strong negative currents. The colors in the trajectories indicate the speed of the particle.

estimated timescale of $t_{\|} \approx 0.09 t_{0}$ corresponds to what is observed in the particle simulations for the electrons to travel a distance $L$ in the parallel direction (see Fig. 3 ).

In this picture, electrons that find strong current channels acquire a higher parallel speed (and travel a distance $L$ sooner). This is confirmed by Figure 7, which shows the trajectory of three of the highest speed electrons. The structures in black and white correspond to strong negative and positive currents (they are surface contours of the parallel current). The trajectories of the electrons are indicated by the thick lines, with changing colors corresponding to increasing speed of the electron. It is quite clear in this figure that these high-speed electrons have found a current channel (two of them found a strong negative current, so they move up, while the other one found a strong positive current, so it moves down) and that they move essentially along the direction of the strong magnetic field. That is, electrons are essentially magnetized and attain high parallel speeds in strong current channels.

The maximum current can also be estimated to be $J_{\|}^{\max }=$ $\delta B / \rho_{i i}$. Then, $J^{\max } / J_{0}=L / \rho_{i i}$. Using equation (13), we can write the maximum parallel energy $\epsilon_{\|}^{\max }=m_{e} u_{\|}^{2} / 2$ of an electron by this mechanism as

$$
\epsilon_{\|}^{\max }=\left(\frac{L}{\rho_{i i}}\right)^{2} \frac{1}{R_{m}} m_{p} v_{0}^{2} .
$$

In principle, electrons in the current channels will see a weak transverse magnetic field $\boldsymbol{b}_{\perp}$ (the reconnecting field inside the current sheet); however, as we pointed out the reconnection geometry observed in turbulent simulations is not ideal, and eventually the particles will see a transverse magnetic field than can be large. At that point, the electron with a large parallel velocity will pitch-angle scatter and some of the energy will then be transferred to the perpendicular velocity. This is the role of the terms containing $b_{\perp}$ in the transverse and parallel equations of motion for the electrons that we have ignored before. 
By such mechanism, electrons can gain some perpendicular energy, a fact that it is observed in the velocity distribution plots (see middle and bottom panels of Fig. 5). Perpendicular velocities larger than the plasma speed $v_{0}$ are observed after several electron gyroperiods.

We have identified several ways by which the test electrons can gain energy. In the very initial stage, at timescales of the order of the electron nominal gyroperiod, electrons (if started from rest) will get a perpendicular velocity of the order of the plasma speed $\boldsymbol{v}_{\perp}$. On a longer timescale $t_{\|}$(but still small compared to the MHD timescale $t_{0}$ ), some electrons get large parallel speeds due to motion along current channels, until they travel a correlation length $L$ (or leave the region in consideration). A third stage consists of some transfer of energy to perpendicular motion, due to pitch-angle scatter effects from the transverse magnetic field fluctuations $\boldsymbol{b}_{\perp}$. There is another possibility by which particles can gain energy, but we have ignored so far this effect for the electrons. This comes from the particles moving in the turbulent velocity field $\boldsymbol{v}_{\perp}$, which is far from uniform. In this case particles could get energy by sampling the variations of the velocity field (which implies that particles see a nonuniform electric field and can be subject to kicks aligned with their velocity). The reason for ignoring this effect for the electrons is their small gyroradius, compared with the MHD turbulence scales. To support this fact, we show in Figure 8 the distribution of electron gyroradii, computed as $r_{g}^{e}=u_{\perp} m_{e} c /\left(e B_{0}\right)$.

The arrow in the plot indicates the dissipation length of the turbulence (i.e., the smallest resolved MHD scale), which we have set to be $l_{d} \sim \rho_{i i}$. It can be seen that the electron gyroradii are much smaller than the smallest resolved MHD scale in this simulation.

Since electrons are then gyrating with high frequency and small gyroradius, they essentially see the same fields for many gyroperiods and there is no substantial energy gain by this effect (kicks by the electric field are first aligned, then antialigned with the particle velocity in rapid fashion). As we see in the next section, this situation changes completely for the case of protons.

\subsection{Protons}

Considering $m=m_{p}, Z=1, \rho_{i i} / L=1 / 32$, and $\delta B / B_{0}=$ $1 / 10$, then $r_{0}^{p} / L \approx 3.1 \times 10^{-3}$. Here $r_{0}^{p}$ is the nominal gyroradius $r_{0}$ (see eq. [5]) for the particular case of protons.

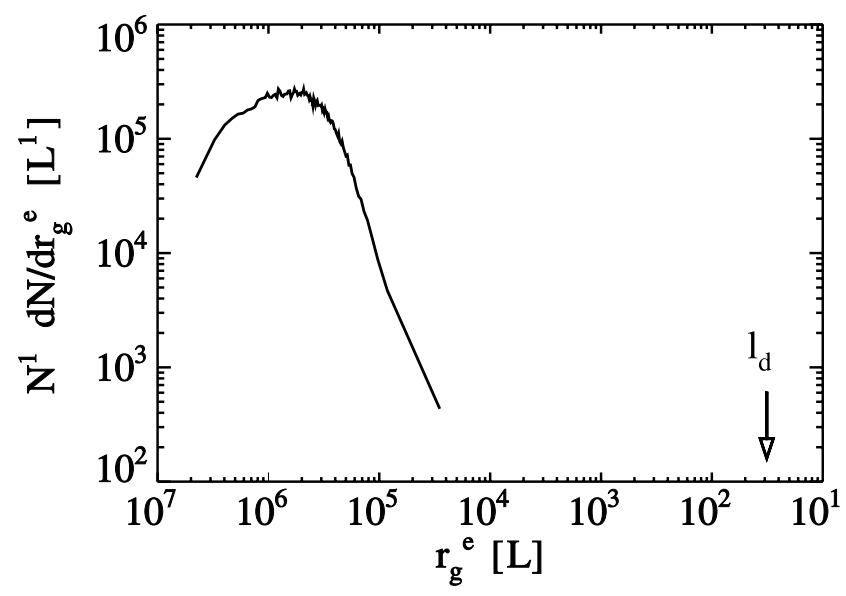

FIG. 8.-Distribution function of electron gyroradii $r_{g}^{e}$ at $t=10^{4} \tau_{e}=$ $0.09 t_{0}$. The electron gyroradius is defined as $r_{g}^{e}=u_{\perp} m_{e} c /\left(e B_{0}\right)$, with $u_{\perp}$ the electron perpendicular velocity. The arrow indicates the MHD dissipation scale $l_{d} \approx \rho_{i i}$.
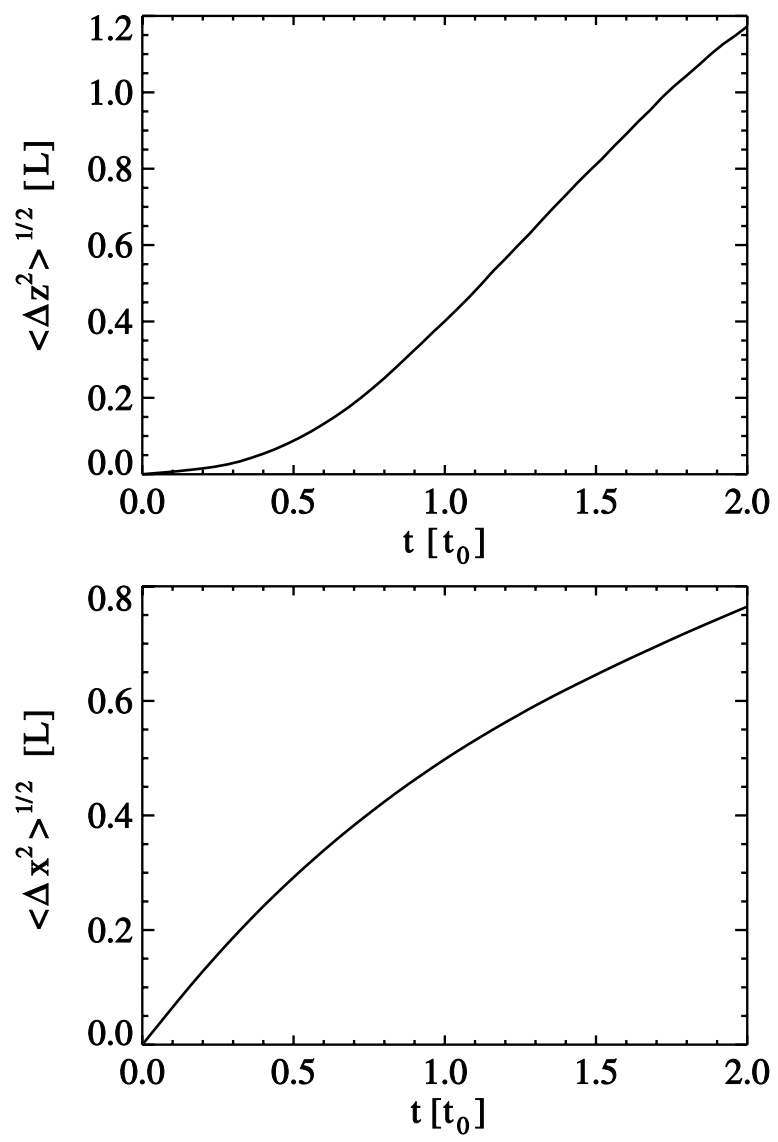

FIG. 9.-Top: The rms displacement of protons as a function of time in the $z$-direction (parallel to the background magnetic field) $\left\langle\Delta z^{2}\right\rangle^{1 / 2}$. Bottom: The rms displacement in a transverse direction $\left\langle\Delta x^{2}\right\rangle^{1 / 2}$.

The rms displacement of protons as a function of time in the parallel $(z)$ and a transverse direction (it does not make any difference which transverse direction we choose, $x$ or $y$ ) is shown in Figure 9. We see again that the parallel displacement is larger, but not as much as in the case of electrons. The average timescale to travel a distance $L$ in the parallel direction is now $t \approx 1.8 t_{0}$, much larger than in the case of electrons. A sharp contrast with the electrons results is in Figure 10, showing the mean square parallel and transverse velocities as a function of time. There is a large gain in the transverse velocity direction, reaching an rms value $\left\langle\Delta u_{x}^{2}\right\rangle^{1 / 2} \sim 15 v_{0}$ in $t \approx 1.8 t_{0}$. The ratio of a mean square transverse velocity and the mean square parallel velocity maintains a high value throughout, with a value of $\left\langle\Delta u_{x}^{2}\right\rangle /\left\langle\Delta u_{z}^{2}\right\rangle \approx 25$ after an initial decrease (see Fig. 10). This implies the same ratio of perpendicular to parallel temperature for protons, if defined as proportional to the mean square velocities.

The evolution of the velocity distribution function of protons at three different times can be seen in Figure 11. The top panel corresponds to $t=0.04 t_{0}$, approximately equal to $2 \tau_{p}$, where $\tau_{p}=2 \pi m_{p} c /\left(e B_{0}\right)$ is the proton gyroperiod. The distribution function of parallel velocities $u_{z}$ of protons is indicated with the solid line, while the dashed line indicates the distribution of the transverse velocities $u_{x}$ (essentially identical to the distribution of $u_{y}$, not shown). Values of the order of the plasma speed $v_{0}$ can be observed for the transverse velocity already at this short time. The middle panel shows the distributions at $t=0.4 t_{0}=20 \tau_{p}$. Here a noticeable increase of the transverse velocities is observed. The bottom panel shows the 

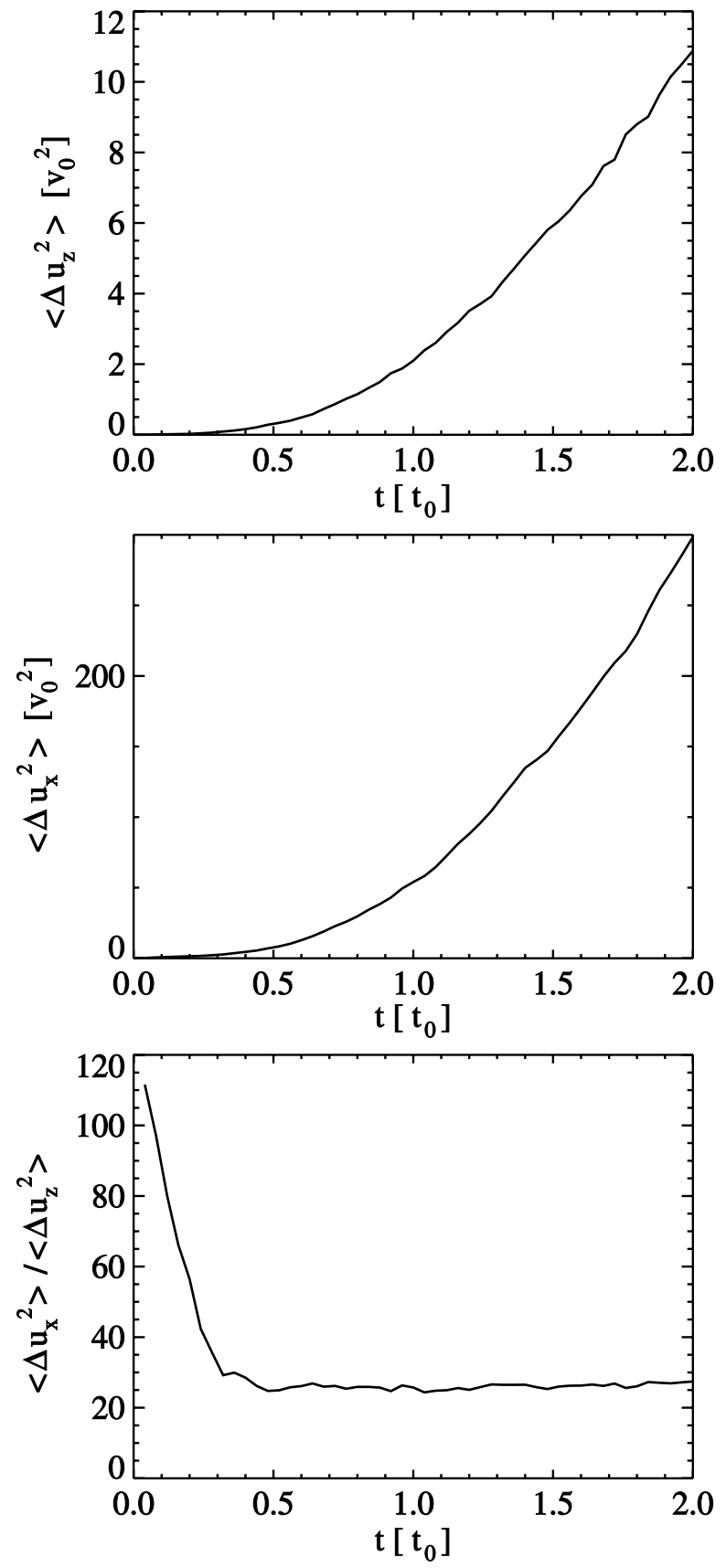

FIG. 10.-Top: Mean square velocity of protons as a function of time in the parallel direction $\left\langle\Delta u_{z}^{2}\right\rangle$. Middle: Mean square velocity in a transverse direction $\left\langle\Delta u_{x}^{2}\right\rangle$. Bottom: Ratio of transverse to parallel mean square velocities $\left\langle\Delta u_{x}^{2}\right\rangle /\left\langle\Delta u_{z}^{2}\right\rangle$.

distributions at the final time in this simulation, $t=1.8 t_{0}=$ $90 \tau_{p}$. A substantial increase in the transverse velocities of protons is seen here. Parallel velocities have increased as well, but a large ratio of transverse to parallel velocities is observed at the large velocity tails and is somehow smaller at the core part of the distributions. The scatter plots of proton velocities in Figure 12 show clearly the anisotropic behavior. The left panel shows the parallel $u_{z}$ and transverse velocity $u_{x}$, while the right panel shows the two transverse components $u_{x}$ and $u_{y}$.

We attempt now to understand qualitatively the behavior of protons by the effect of the different terms in the equations of motion, as we have done for the electrons in the previous
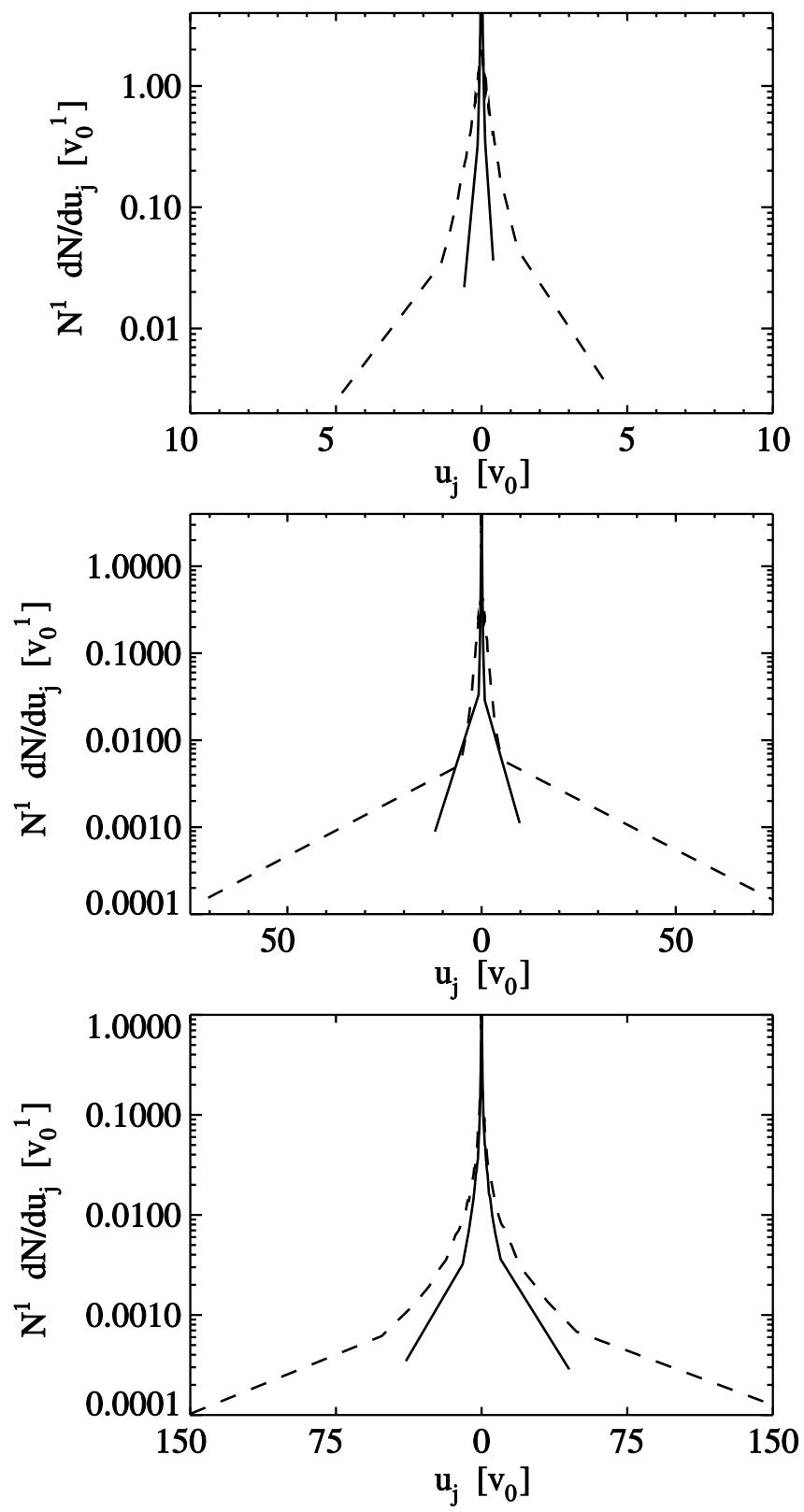

FIG. 11.-Velocity distribution functions of protons at different times. The continuous line is the distribution of velocities in the parallel direction, and the dashed line is the distribution of velocities in a transverse direction. Top: Distributions at $t=2 \tau_{p}=0.04 t_{0}$. Middle: Distributions at $t=20 \tau_{p}=0.4 t_{0}$. Bottom: Distributions at $t=90 \tau_{p}=1.8 t_{0}$.

section. The equations for the transverse and parallel velocity of the protons are given by (using $q=e$ and $m=m_{p}$ )

$$
\begin{gathered}
\frac{d \boldsymbol{u}_{\perp}}{d t}=\frac{e}{m_{p} c}\left[\left(\boldsymbol{u}_{\perp}-\boldsymbol{v}_{\perp}\right) \times \boldsymbol{B}_{\|}+\left(\boldsymbol{u}_{\|}-\boldsymbol{v}_{\|}\right) \times \boldsymbol{b}_{\perp}+\frac{v_{0} L}{R_{m}} \boldsymbol{J}_{\perp}\right] \\
\frac{d \boldsymbol{u}_{\|}}{d t}=\frac{e}{m_{p} c}\left[\left(\boldsymbol{u}_{\perp}-\boldsymbol{v}_{\perp}\right) \times \boldsymbol{b}_{\perp}+\frac{v_{0} L}{R_{m}} \boldsymbol{J}_{\|}\right] .
\end{gathered}
$$

As for electrons, the first term on the right-hand side of equation (15) describes a drift motion with velocity $\boldsymbol{v}_{\perp}$ plus a gyromotion around the magnetic field $\boldsymbol{B}_{\|} \approx B_{0} \hat{z}$. This 

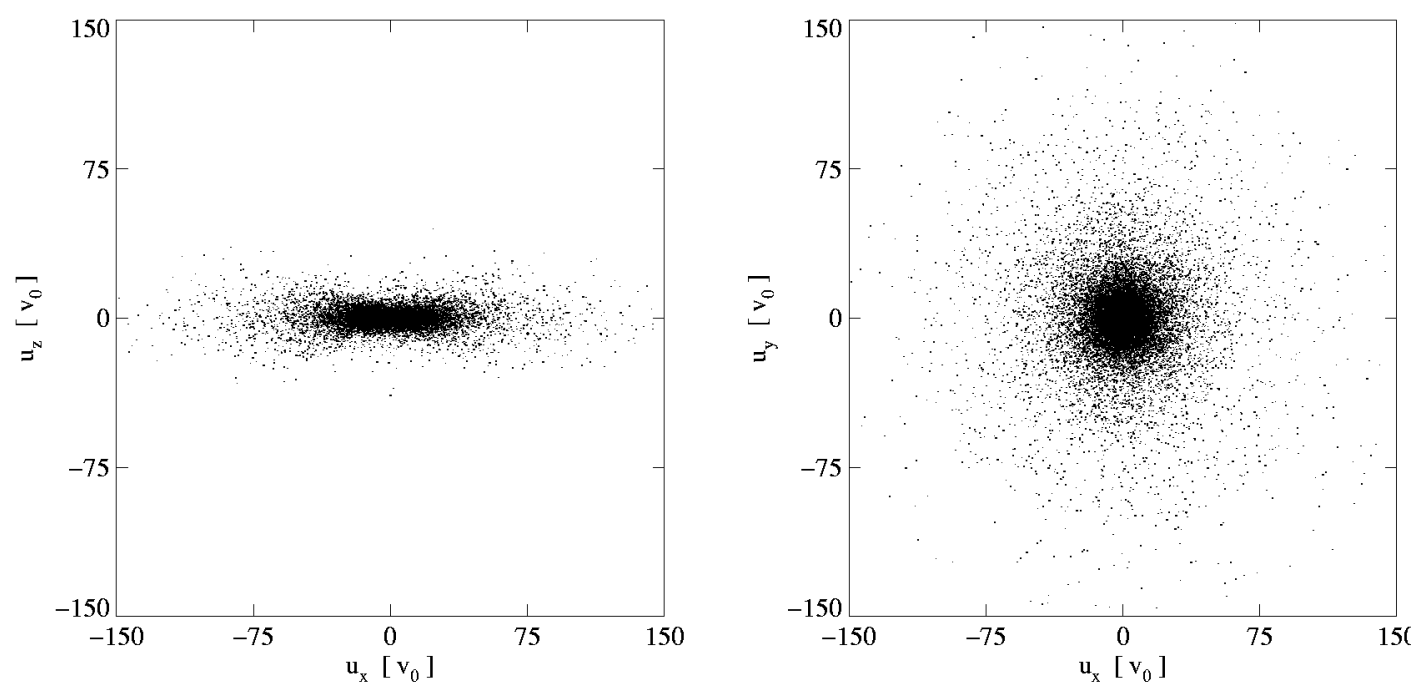

Fig. 12. - Left: Scatter plot of transverse $\left(u_{x}\right)$ and parallel $\left(u_{z}\right)$ velocity of protons. Right: Scatter plot of transverse velocities $\left(u_{x}, u_{y}\right)$.

drift + gyromotion occurs here on a timescale of order $\tau_{p}=$ $2 \pi m_{p} c /\left(e B_{0}\right)$, which as we have seen corresponds to $t \approx$ $0.02 t_{0}$. So on a short timescale (compared to the MHD timescale $t_{0}$ ) protons acquire a transverse velocity of the order of the transverse plasma velocity $\boldsymbol{v}_{\perp}$ at that location. This is supported by the results of the transverse velocity distribution function of protons in the top panel of Figure 11, which corresponds to $t \approx 2 \tau_{p}$. The difference in mass of protons and electrons of course implies that the timescale of this initial stage is much larger than the corresponding timescale for electrons, $\tau_{p} \gg \tau_{e}$. More importantly, the fact that the gyroradius of electrons was much smaller than the MHD dissipation scale (of the order of the current sheet thickness) allowed them to remain inside the current channels over several gyroperiods. For the protons, on the other hand, the gyroradius is much larger and comparable initially to the current sheet thickness. Protons, then, do not remain inside the current channels and they do not see a coherent parallel current, which could produce a net energization effect, as happens for the electrons.

The fact that the proton gyroradius is initially of the order of the current sheet thickness (the MHD dissipation length scale) and grows in time is shown in Figure 13. The distribution function of proton gyroradii, obtained as $r_{g}^{p}=u_{\perp} m_{p} c /\left(e B_{0}\right)$, is shown at three different times in the evolution. The values are clearly increasing with time as a result of the increase in the transverse velocity $u_{\perp}$ of protons, which has already been shown in the previous plots.

Where might this energization in the transverse direction come from? Unlike the electrons, whose small gyroradius means that they essentially see the same fields over many gyroperiods, protons, with gyroradius of the order of the MHD dissipation scale, may sample variations of the fields that are in phase with their own velocity. As a result, a fraction of the protons may increase their energy considerably. Physically what happens is that protons see a transverse MHD-induced electric field $\boldsymbol{E}_{\perp}=-\left(\boldsymbol{v}_{\perp} \times B_{0} \hat{z}\right) / c$ that varies on scales comparable to the proton gyroradius. If kicks of this electric field are in phase with the proton velocity, this results in a net increase of the perpendicular energy. Variations of the transverse electric field are actually produced by variations in the plasma velocity $\boldsymbol{v}_{\perp}$. The effect of these variations can be directly seen through the proton equations of motion. To do that, we consider the derivative of the plasma velocity "following a particle trajectory"; this is

$$
\frac{d \boldsymbol{v}_{\perp}}{d t}=\boldsymbol{u} \cdot \nabla \boldsymbol{v}_{\perp} \approx \boldsymbol{u}_{\perp} \cdot \nabla_{\perp} \boldsymbol{v}_{\perp}
$$

the latter approximation as a result of both large transverse proton velocity compared to the parallel one and small variations of the plasma velocity along the magnetic field direction. Subtracting equation (17) from equation (15) results in

$$
\begin{aligned}
\frac{d\left(\boldsymbol{u}_{\perp}-\boldsymbol{v}_{\perp}\right)}{d t}= & \frac{e}{m_{p} c}\left[\left(\boldsymbol{u}_{\perp}-\boldsymbol{v}_{\perp}\right) \times \boldsymbol{B}_{\|}+\left(\boldsymbol{u}_{\|}-\boldsymbol{v}_{\|}\right) \times \boldsymbol{b}_{\perp}+\frac{v_{0} L}{R_{m}} \boldsymbol{J}_{\perp}\right] \\
& -\boldsymbol{v}_{\perp} \cdot \nabla_{\perp} \boldsymbol{v}_{\perp}-\left(\boldsymbol{u}_{\perp}-\boldsymbol{v}_{\perp}\right) \cdot \nabla_{\perp} \boldsymbol{v}_{\perp} .
\end{aligned}
$$

The last term on the right-hand side implies that if variations of the plasma transverse velocity field are in phase with the gyromotion of the particle (represented here by the difference $\boldsymbol{u}_{\perp}-\boldsymbol{v}_{\perp}$ between the particle velocity $\boldsymbol{u}_{\perp}$ and the drift $\boldsymbol{v}_{\perp}$ due to the plasma), then an energy increase can be obtained through this motion. This energy increase might be exponential in an ideal resonant-like situation. The rate at which this energy increase proceeds could be estimated by the gradient of the plasma speed $\nabla_{\perp} v_{\perp} \sim v_{0} / r$, where $r$ is the proton gyroradius. At the same time, the increase in the transverse velocity increases the gyroradius, so the gradients in the fields seen by the particle become smaller and the energy gain slows down. To complicate the picture even more, at some point the transverse velocity of the proton is very high, and the pitch-angle scattering effects due to the transverse magnetic field fluctuations $\boldsymbol{b}_{\perp}$ come into play. The parallel velocity of the protons can then increase because of the term $\left(\boldsymbol{u}_{\perp}-\boldsymbol{v}_{\perp}\right) \times \boldsymbol{b}_{\perp}$ in equation (16). This increase in the parallel velocity of protons is observed in the distribution function plots shown in Figure 11. There is then an observable motion in the parallel direction, and eventually protons may travel a correlation length $L$ or leave the region in consideration, at which point the energy gain due to the previously described resonant-like process stops.

The complex motion of the protons can be seen in Figure 14, which shows the trajectories of three of the most energetic 

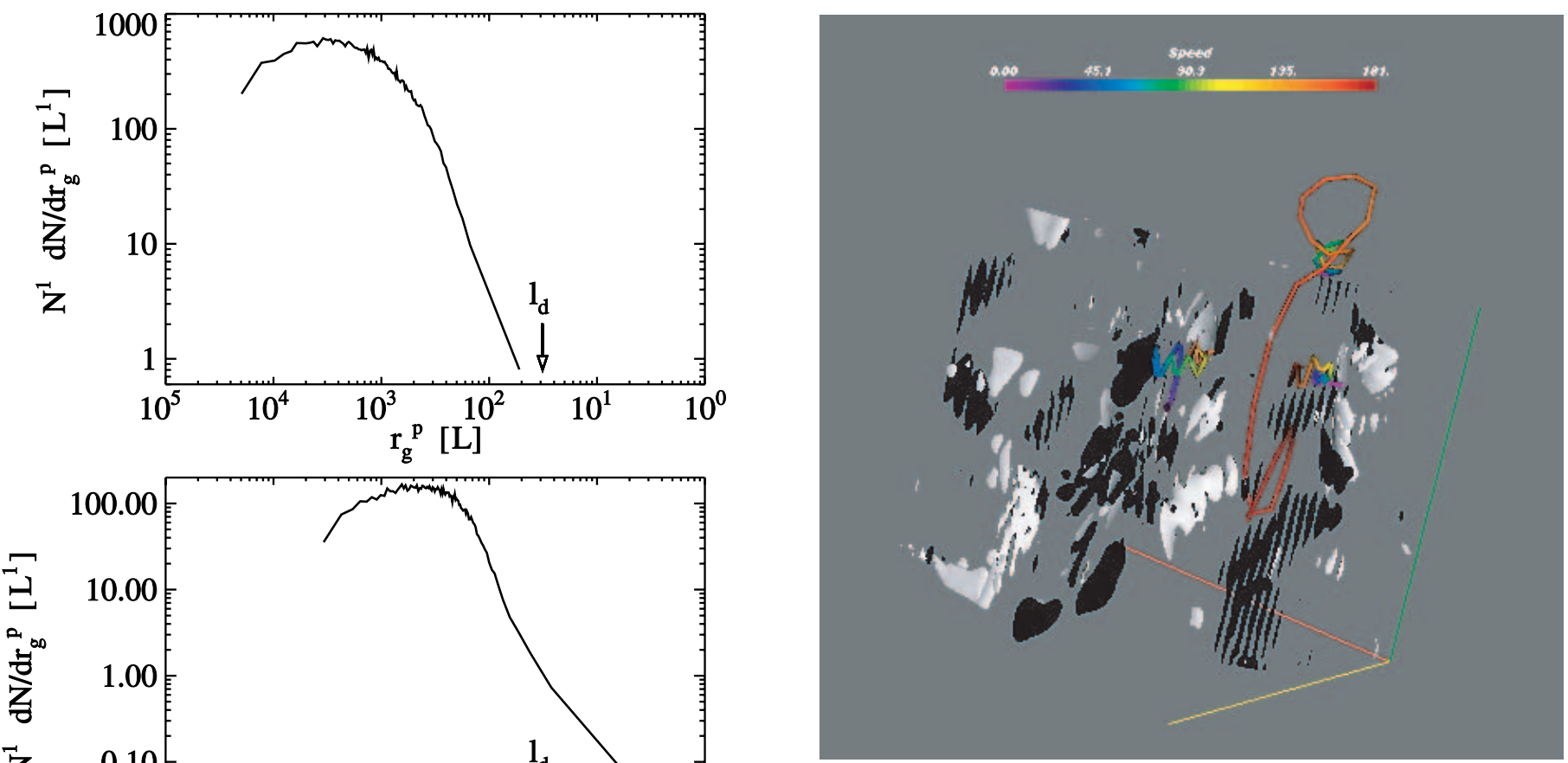

FIG. 14.-Trajectories of three of the most energetic protons. The structures in the background are contour surfaces of the current density $J_{z}$, with white indicating strong positive currents and black strong negative currents. The colors in the trajectories indicate the speed of the particle.

of dimensionless quantities as $\left(u_{\perp} / v_{0}\right)=\left(B_{0} / \delta B\right)\left(r / \rho_{i i}\right)$. The problem here is to determine what is the critical gyroradius at which the energy gain process stops. Since we do not have a simple analytical model for the motion of the most energetic protons, as we did for the electrons, we can just give an estimate. According to the scenario that we showed for the protons, this critical gyroradius $r$ could take values between $L$ and $\rho_{i i}$. Assuming a value given by the geometric mean $\left(L \rho_{i i}\right)^{1 / 2}$, we can estimate that the maximum perpendicular speed acquired by a proton is

$$
\frac{u_{\perp}}{v_{0}} \sim \frac{B_{0}}{\delta B}\left(\frac{L}{\rho_{i i}}\right)^{1 / 2} .
$$

FIG. 13.-Distribution function of proton gyroradii $r_{g}^{p}$ at three different times. Top: $t=2 \tau_{p}=0.04 t_{0}$. Middle: $t=20 \tau_{p}=0.4 t_{0}$. Bottom: $t=90 \tau_{p}=$ $1.8 t_{0}$. The proton gyroradius is defined as $r_{g}^{p}=u_{\perp} m_{p} c /\left(e B_{0}\right)$, with $u_{\perp}$ the proton perpendicular velocity. The arrows indicate the MHD dissipation scale $l_{d} \approx \rho_{i i}$.

protons in this simulation. Unlike the electrons, the motion of the protons cannot be associated here with the presence of strong current channels (indicated with white and black contour surfaces for reference). One of the proton trajectories shows the pitch-angle scattering effect (an increase in the parallel motion) mentioned before. In all three trajectories, the gyromotion on scales of the turbulent fields can be observed. This gyromotion with an increase in the gyroradius as the particle velocity increases can be clearly seen in Figure 15, which shows the same trajectories of the most energetic protons, but now seen from the top.

The perpendicular speed of the protons can be related to the gyroradius by $u_{\perp}=w_{p} r$, where $w_{p}=\left(e B_{0}\right) /\left(m_{p} c\right)$ is the nominal proton gyrofrequency. This relation can be put in terms

Using the values that we assumed for protons, we get $u_{\perp} \approx 60 v_{0}$, which falls short of the result obtained in the simulations. This can be "corrected" by assuming a somewhat larger critical gyroradius of $r=4\left(L \rho_{i i}\right)^{1 / 2}$. The relation given by equation (19) should actually be thought of as a scaling to estimate only the order of magnitude of the maximum perpendicular speed of protons.

The maximum perpendicular energy of protons can be obtained as $\epsilon_{\perp}^{\max }=m_{p} u_{\perp}^{2} / 2$, so then the estimation is

$$
\epsilon_{\perp}^{\max } \sim\left(\frac{B_{0}}{\delta B}\right)^{2} \frac{L}{\rho_{i i}} \frac{1}{2} m_{p} v_{0}^{2} .
$$

\section{DISCUSSION}

By doing test particle simulations we have shown different mechanisms by which electrons and protons can gain energy in a MHD turbulent plasma. Two main ingredients have been 


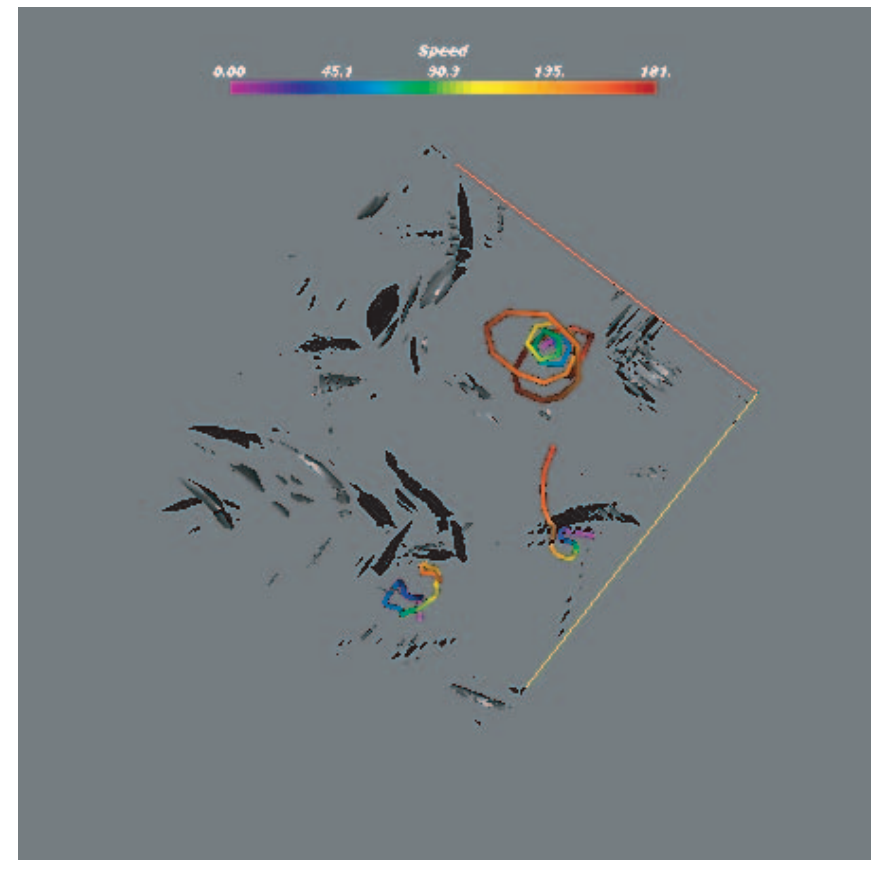

FIG. 15.-Trajectories of three of the most energetic protons. Same plot as in Fig. 14 but viewed from the top of the simulation box.

assumed for the MHD fields: the presence of a strong magnetic field $B_{0} \hat{z}$, which implies formation of parallel current channels (as a result of the MHD evolution), and the assumption that MHD dissipation scales are of the order of the ion inertial scale $\rho_{i i}$. A very different behavior of electrons and protons was found in that situation.

The conditions that we assumed for the MHD fields are probably found in realistic astrophysical situations. That is the case for instance in the solar wind, where measurements of the magnetic energy spectra have allowed identification of the MHD dissipation scale with the ion inertial scale (Leamon et al. 1998) and could certainly be found in a coronal hole (lower corona), where both assumptions of a strong magnetic field and dissipation scales are highly plausible. To this end, we could expect that the effects we have seen should be present in the coronal plasma. That provides an interesting alternative for effective dissipation mechanisms in coronal models. From other research that we have done (Dmitruk \& Matthaeus 2003), we believe that the MHD structures that we see here could be present, for instance, in coronal holes. In that case, energy injected in the form of waves is transferred to small perpendicular scales through anisotropic turbulence. The direct particle acceleration processes shown here could then provide a channel for the actual dissipation of the energy that reached the small scales. Interestingly, large parallel velocities for electrons and large transverse velocities for protons (and other heavy particles), which we showed here, is what is widely believed to be consistent with observations in the corona (Kohl et al. 1997, 1998).

The limitation on the resolution of the possible length scales is perhaps the main obstacle in the direct approach that we took (in contrast to modeling the turbulence), if we want to apply the present simulations to astrophysical situations. The scalings that we obtained could, however, be extrapolated to the larger values present in those situations. Another limitation that we have to face (if we intend to consider this as a dissipation mechanism for a coronal heating model) is the lack of self-consistency (particles do not feed back into the MHD fields). At some point, the energy gain process may slow down, not only because of escaping effects, as we considered, but also because of particles producing waves and instabilities in the fields. The effects of collisions should also be taken into account. Including these effects but still working with a test particle simulation approach requires further research on the subject (possibly through modeling the energy loss processes; see for instance a recent paper by Ivlev et al. 2004).

At present we are currently working on two improvements that are more straightforward to achieve. One is considering the relativistic equations of motion for the particles, necessary when large astrophysical values are assumed. The other one is including the Hall effect modification to the MHD equations. This is a correction to the Ohm law for the induced electric field, which can be important at length scales comparable to the ion inertial scale (Krall \& Trivelpiece 1973). Preliminary results, however, show that this has no important effect during the particle energization process in the setting that we have assumed with a strong magnetic field. This is due to the fact that the extra term given by the Hall effect in the electric field is mainly in the perpendicular direction, where it only produces a modification of the drift speed acquired by particles at the initial stage. Detailed results are going to be shown in a further publication, to keep the present study simpler.

\section{CONCLUSION}

We performed test particle simulations in turbulent fields obtained from direct numerical solutions of compressible three-dimensional MHD with a strong background magnetic field. The natural tendency of the turbulent MHD fields is to form singular structures (current sheets) along the magnetic field direction, as well as strong nonuniform fields in the transverse directions. A key feature was to associate the MHD dissipation length scale to be of the order of the ion inertial scale. By doing that we found differential energization in parallel and perpendicular directions to the magnetic field according to the type of particles considered.

Electrons, with gyroradius much smaller than the MHD dissipation length scale, develop large parallel velocities, due to the coherent action of the parallel electric field in current channels. In the present case this parallel electric field is due to the ohmic term. In collisionless applications other kinetic effects would presumably supply an alternative electric field of similar magnitude to the ohmic field of the present study. Protons, on the other hand, with gyroradius comparable to or larger than the MHD dissipation length scale, show higher perpendicular energization, due to the nonuniform perpendicular induced electric field produced by the plasma MHD velocity, which varies on proton length scales.

The results shown, although simplified and with the limitations mentioned in the previous section, indicate a promising way of studying some basic issues in the complex topic of bridging the MHD and kinetic descriptions of a plasma.

This research was supported by NASA grant NAG5-7164 and NSF grant ATM 99-77692. The runs were performed on the Beowulf clusters at Bartol Research Institute, University of Delaware. P. D. acknowledges useful conversations with A. A. van Ballegooijen, B. J. Vasquez, and A. R. Barakat at a conference. 
Achatz, U., Steinacker, J., \& Schlickeiser, R. 1991, A\&A, 250, 266

Ambrosiano, J., Matthaeus, W. H., Goldstein, M. L., \& Plante, D. 1988, J.

Geophys. Res., 93, 14383

Arzner, K., \& Vlahos, L. 2004, ApJ, 605, L69

Birn, J., \& Hesse, M. 1994, J. Geophys. Res., 99, 109

Birn, J., Hesse, M., \& Schindler, K. 1989, J. Geophys. Res., 94, 241

Biskamp, D., \& Welter, H. 1989, Phys. Fluids B, 1, 1964

Cranmer, S. R., \& van Ballegooijen, A. A. 2003, ApJ, 594, 573

Dmitruk, P., \& Matthaeus, W. H. 2003, ApJ, 597, 1097

Dmitruk, P., Matthaeus, W. H., Seenu, N., \& Brown, M. R. 2003, ApJ, 597, L81

Gary, S. P., \& Nishimura, K. 2004, J. Geophys. Res., 109, 2109

Giacalone, J., \& Jokipii, J. R. 1999, ApJ, 520, 204

Gosling, J. T., de Koning, C. A., Skoug, R. M., Steinberg, J. T., \& McComas, D. J. 2004, J. Geophys. Res., 109, 5102

Hall, D. E., \& Sturrock, P. A. 1967, Phys. Fluids, 10, 2620

Heerikuisen, J., Litvinenko, Y. E., \& Craig, I. J. D. 2002, ApJ, 566, 512
REFERENCES

Hollweg, J., \& Isenberg, P. 2002, J. Geophys. Res., 107, 1147

Ivlev, A. V., Khrapak, S. A., Zhdanov, S. K., Morfill, G. E., \& Joyce, G. 2004, Phys. Rev. Lett., 92, 205007

Kohl, J. L., et al. 1997, Sol. Phys., 175, 613 1998, ApJ, 501, L127

Krall, N. A., \& Trivelpiece, A. W. 1973, Principles of Plasma Physics (New York: McGraw-Hill)

Leamon, R. J., Smith, C. W., Ness, N. F., Matthaeus, W. H., \& Wong, H. K. 1998, J. Geophys. Res., 103, 4775

Matthaeus, W. H., \& Lamkin, S. L. 1986, Phys. Fluids, 29, 2513

Miller, J. A., et al. 1997, J. Geophys. Res., 102, 14631

Oughton, S., Priest, E. R., \& Matthaeus, W. H. 1994, J. Fluid Mech., 280, 95 Schlickeiser, R., \& Miller, J. A. 1998, ApJ, 492, 352

Schopper, R., Birk, G. T., \& Lesch, H. 1999, Phys. Plasmas, 6, 4318

Shebalin, J. V., Montgomery, D. C., \& Matthaeus, W. H. 1983, J. Plasma Phys., 29,525 